\title{
Antoine TINE
}

Docteur en science politique de l'Institut d'Études politiques de Paris.

Professeur, UFR sciences juridiques et politiques,

Université Gaston Berger, Saint-Louis, Sénégal

(2002)

\section{“《E PLURIBUS UNUM». \\ Essai d'une philosophie politique du pluralisme démocratique."}

Un document produit en version numérique par Jean-Marie Tremblay, bénévole, professeur de sociologie au Cégep de Chicoutimi

Courriel: jean-marie tremblay@uqac.ca

Site web pédagogique : http://www.uqac.ca/jmt-sociologue/

Dans le cadre de: "Les classiques des sciences sociales" Une bibliothèque numérique fondée et dirigée par Jean-Marie Tremblay, professeur de sociologie au Cégep de Chicoutimi Site web: http://classiques.uqac.ca/

Une collection développée en collaboration avec la Bibliothèque Paul-Émile-Boulet de l'Université du Québec à Chicoutimi

Site web: http://bibliotheque.uqac.ca/ 


\section{Politique d'utilisation de la bibliothèque des Classiques}

Toute reproduction et rediffusion de nos fichiers est interdite, même avec la mention de leur provenance, sans l'autorisation formelle, écrite, du fondateur des Classiques des sciences sociales, Jean-Marie Tremblay, sociologue.

Les fichiers des Classiques des sciences sociales ne peuvent sans autorisation formelle:

- être hébergés (en fichier ou page web, en totalité ou en partie) sur un serveur autre que celui des Classiques.

- servir de base de travail à un autre fichier modifié ensuite par tout autre moyen (couleur, police, mise en page, extraits, support, etc...),

Les fichiers (.html, .doc, .pdf., .rtf, .jpg, .gif) disponibles sur le site Les Classiques des sciences sociales sont la propriété des Classiques des sciences sociales, un organisme à but non lucratif composé exclusivement de bénévoles.

Ils sont disponibles pour une utilisation intellectuelle et personnelle et, en aucun cas, commerciale. Toute utilisation à des fins commerciales des fichiers sur ce site est strictement interdite et toute rediffusion est également strictement interdite.

L'accès à notre travail est libre et gratuit à tous les utilisateurs. C'est notre mission.

Jean-Marie Tremblay, sociologue

Fondateur et Président-directeur général, LES CLASSIQUES DES SCIENCES SOCIALES. 
Cette édition électronique a été réalisée par Jean-Marie Tremblay, bénévole, professeur de sociologie au Cégep de Chicoutimi à partir de :

Antoine TINE

[Docteur en science politique de l'Institut d'Études politiques de Paris.]

“«E PLURIBUS UNUM ». Essai d'une philosophie politique du pluralisme démocratique".

Conférence donnée au Consortium de philosophie de Saint-Augustin, Dakar : 2002. Centre Saint Augustin, Cahier de Réflexion, n8, 2002-2003.

[Autorisation formelle accordée par l'auteur le 2 avril 2008 de diffuser ce texte dans Les Classiques des sciences sociales.]

91 Courriels : tony tine66@yahoo.fr ou antoinetine@hotmail.com

Polices de caractères utilisée :

Pour le texte: Times New Roman, 14 points.

Pour les citations : Times New Roman, 12 points.

Pour les notes de bas de page : Times New Roman, 12 points.

Édition électronique réalisée avec le traitement de textes Microsoft Word 2004 pour Macintosh.

Mise en page sur papier format : LETTRE (US letter), 8.5', x 11'’)

Édition numérique réalisée le 18 avril 2008 à Chicoutimi, Ville de Saguenay, province de Québec, Canada. 


\section{Table des matières}

Résumé

Problématique

1. À travers la science politique

2 Philosophie du pluralisme: entre l'un et le multiple

\section{Bibliographie}


Antoine TINE

“«E PLURIBUS UNUM ». Essai d'une philosophie politique du pluralisme démocratique".

Conférence donnée au Consortium de philosophie de Saint-Augustin, Dakar : 2002. Centre Saint Augustin, Cahier de Réflexion, n8, 2002-2003.

«Une société n'est pas un corps unitaire dans lequel s'exercerait un pouvoir et seulement un, mais c'est en réalité une juxtaposition, une liaison, une coordination, une hiérarchie aussi, de différents pouvoirs, qui néanmoins demeurent dans leur spécificité $\gg$.

Michel FOUCAULT « Les mailles du pouvoir » in Dits et Ecrits 1954-1988. Tome IV : 1980-1988, Paris, Gallimard, 1994, p. 187.

«L'État est l'un, auquel est ordonné le multiple. Il est ce par quoi s'unifie ce qui ne serait qu'un conglomérat inconsistant de "peuplades", de "populations", ce qui enfin donne forme et partant valeur à un magma sans qualités, à une matière première, un quasinéant ou pure négativité ».

Fabien EBOUSSI BOULAGA, Les conférences nationales en Afrique noire. Une affaire à suivre, Paris, Karthala, 1993, p. 99.

\section{RÉSUMÉ}

On tient, depuis Hannah Arendt, que la philosophie politique est le domaine de la pluralité, à la fois pluralité des hommes, des intérêts et des opinions. Plus précisément, le passage du parti unique au multipartisme représente l'exigence de l'invention démocratique, une sorte de dramatique de l'événement fondateur et une liturgie de l'initiation démocratique, c'est-à-dire "passage à la maturité et à la pleine res- 
ponsabilité sociale », « alliance avec des droits et des obligations réciproques » entre membres d'une même collectivité politique, instituant un style de vie ou un ethos. Le mythe politique du pluralisme démocratique n'est pas nécessairement mensonge, mais « faire croire » instaurant une légitimité fondatrice et se révélant comme un ensemble de procédures et d' " arts de faire » et une « invention paradoxale de la modernité ». Dans la pensée politique contemporaine, la question de la démocratie pluraliste soulève le problème des rapports entre l'un et le multiple, entre l'absolu et le relatif, entre les ruses de la logique unitaire et les conflits des opinions contradictoires, ou la " contradiction potentielle entre le vivre-ensemble des citoyens et leur hétérogénéité ». Le pluralisme démocratique doit être relié à l'exigence de consensus et d'unité, car il n'y a pas de société démocratique sans volonté de vivre ensemble, sans un accord raisonnable sur les règles du jeu politique. Le pluralisme est certes constitutif de l'histoire des partis politiques, mais la problématique politique est gouvernée par les exigences de cohérence et d'unité. Le pluralisme lui-même ne se donne t-il pas comme une "idée maîtresse » unique, comme " une grande chose $» ?$

Summary: It has been accepted, since Hannah Arendt, that political philosophy is the sphere of plurality, at the same time plurality of men, interests and opinions. More precisely, the transition from unique party to multipartism represents the requirement of democratic invention, a kind of drama of founding event and a liturgy of democratic initiation, that is "transition to maturity and to complete social responsibility", "alliance with rights and reciprocal obligations" among the members of the same political group. That institutes a life style or an "ethos". The political myth of democratic pluralism is not necessarily a lie but a hope creating legitimate foundation and revealing itself as a whole of procedures and "arts of doing" and a "paradoxical invention of modernity". In the contemporary political thought, the question of pluralist democracy raises the problem concerning the relationships between the One and the Multiple, between what is absolute and what is relative, between the trickeries of the uni- 
tary logic and the conflicts of contradictory opinions, or the "potential contradiction between the living-together of citizens and their homogeneity". The democratic pluralism must be related to the requirement of consensus and unity, because there can't be a democratic society without the will of living together, without any reasonable agreement on the rules of the political game. Pluralism is certainly related the history of political parties, but politics is ruled by the requirements of coherence and unity. Does'nt pluralism present itself as a unique "key idea", as a "great thing"?

\section{PROBLÉMATIQUE}

$\underline{\text { Retour à la table des matières }}$

On tient, depuis Hannah Arendt, que la philosophie politique est le domaine de la pluralité, à la fois pluralité des hommes, des intérêts et des opinions ${ }^{1}$. Plus précisément, le passage du parti unique au multipartisme (pluralisme) a représenté l'exigence de l'invention démocratique, une sorte de dramatique de l'événement fondateur et une liturgie de l'initiation démocratique, c'est-à-dire « passage à la maturité et à la pleine responsabilité sociale $»^{2}$, " alliance avec des droits et des obligations réciproques ${ }^{3}$ entre membres d'une même collectivité politique, instituant un style de vie ou un ethos. Le pluralisme politique est comme un mythe de fondation de la démocratie. Le mythe politique du pluralisme démocratique n'est pas nécessairement mensonge, mais «faire croire » instaurant une légitimité fondatrice et se

1 - Hannah ARENDT, Qu'est-ce que la politique, Paris, Seuil, 1995.

2 Fabien EBOUSSI BOULAGA, Les conférences nationales en Afrique noire. Une affaire à suivre, Paris, Karthala, 1993, p. 159.

3 F. EBOUSSI BOULAGA, Les conférences nationales en Afrique noire, op. cit., p. 160. 
révélant comme un ensemble de procédures et d' " arts de faire ${ }^{4}$ et une « invention paradoxale de la modernité » 5 .

L'expérience politique balance sans cesse entre l'être et le paraître, entre le plein et le vide, entre le sens et le non-sens. Rendre compte de la représentation du pluralisme, c'est proprement envisager l'espace temps de crise du processus de formation de la démocratie. Dans la pensée politique contemporaine, la question de la démocratie pluraliste soulève le problème des rapports entre l'un et le multiple, entre l'absolu et le relatif, entre les ruses de la logique unitaire et les conflits des opinions contradictoires, ou la " contradiction potentielle entre le vivre-ensemble des citoyens et leur hétérogénéité » ${ }^{6}$. Le pluralisme démocratique doit être relié à l'exigence de consensus et d'unité, car il n'y a pas de société démocratique sans volonté de vivre ensemble, sans un accord raisonnable sur les règles du jeu politique :

«(...) la démocratie, cette autre manière de dire le pluralisme, est toujours tendue vers la recherche de l'unité ${ }^{7}$.

Dans son essai sur Guerre et Paix de Tolstoï ${ }^{8}$, Isaiah BERLIN divise les penseurs en deux catégories : 1)- les « hérissons » qui sont

4 Michel DE CERTEAU, L'Invention du quotidien. Tome I : Arts de faire, Paris, UGE, 1980.

5 Jean-François BAYART, L'illusion identitaire, Paris, Fayard, 1989, pp. 231248.

6 - Nicolas TENZER, Philosophie politique, Paris, PUF, $1998^{2}$, p. 18.

7 Jean-Marie DONEGANI et Marc SADOUN, La démocratie imparfaite. Essai sur le parti politique, Fayard, 1994, pp.11-12.

8 Isaiah BERLIN, Les penseurs russes, Albin Michel, 1984; (avec Bernard WILLIAMS), «Pluralism and Liberalism : A Reply », in Political Studies, 62, juin 1994, pp. 306-309 ; Sur la pensée politique de Berlin, voir: Gil DELANNOI, « Sur Isaiah Berlin », in Esprit, mars-avril 1989 ; John Gray, Isaiah Berlin, Harper-Collins / Princeton University Press, 1996 ; Jean LECA, « Libéralisme, pluralisme, communautarisme : actualité d'Isaiah Berlin », in Commentaires, 70, 1995, pp. 369-382 et du même auteur : "Isaiah Berlin. Eloge de la liberté », in François CHATELET, Olivier DUHAMEL, Evelyne PISIER, Dictionnaire des oeuvres politiques, Paris, P.U.F, 1995, 106-123. 
cramponnés à une " vision unique, centrale, un système (...), un principe organisateur universel»; 2)- les « renards » qui «poursuivent plusieurs fins, souvent sans liens, sinon contradictoires entre elles ». Pour Berlin, le pluralisme court avec les renards et engage dans une multiplicité d'objets et d'expériences ${ }^{9}$. Mais, l'affirmation du pluralisme exclue t-elle une «interprétation hérisonnienne » de la réalité politique ? Le pluralisme est certes constitutif de l'histoire des partis politiques, mais la problématique politique est gouvernée par les exigences de cohérence et d'unité. Le pluralisme lui-même ne se donne til pas comme une "idée maîtresse » unique, comme « une grande chose $» ?$

Il y a lieu de s'interroger sur la corrélation entre pluralisme et démocratie. Quelles sont les implications et les fondements de la question du pluralisme au regard de la problématique démocratique? Bien loin de faire une histoire des idées politiques autour de cette notion, il s'agit d'esquisser les traits essentiels du pluralisme politique au regard de la problématique démocratique.

9 “ Si les sociétés humaines étaient des systèmes, ce seraient des systèmes autocontradictoires et non dialectisables parce que non réductibles à une seule finalité ou à un seul moteur originel. La ténacité et la séduction des " hérissons ”, qui ne savent qu'une chose mais grande, n’ont jamais convaincu la population des "renards", qui savent la pluralité des choses mais s'emmêlent parfois dans leurs tours parce que l'excès de leur(s) théorie(s) ne peut cacher leur(s) paralogisme(s) et le vide de leur morale et de leur métaphysique (c'est du moins la réponse invariable des hérissons) ": Jean LECA, «La démocratie à l'épreuve des pluralismes », in Revue Française de science politique (46), 2, avril 1996, p. 225. 


\section{1. À travers la science politique...}

\section{$\underline{\text { Retour à la table des matières }}$}

La pensée politique contemporaine est devenue très sensible à la question du pluralisme politique. Dans les années 1950 et 1960, la science politique américaine fut, en quelque sorte, dominée par ce qu'il convient d'appeler les « théories pluralistes » ${ }^{10}$, proches du courant libéral du système politique. Si, comme Weber, l'on cherche une compréhension positive du politique, il semble plus judicieux de se référer à des penseurs comme Montesquieu, Madison, John Stuart Mill, Tocqueville. Plusieurs versions du pluralisme furent développées.

Il ne saurait être question ici de reprendre les différentes nuances du pluralisme. Qu'il suffise ici de souligner comment s'articule d'une façon générale pluralisme et démocratie. Les penseurs du pluralisme politique cherchaient à décrire et à expliquer le fonctionnement réel de la démocratie. Contrairement à beaucoup de libéraux qui insistaient sur l'individualisme citoyen, les pluralistes étaient préoccupés par le «problème des factions » (pluralisme des factions), ou, selon la terminologie moderne, le problème des "groupes d'intérêt» ${ }^{11}$ et des «groupes de pression ». Pour les pluralistes, l'existence des factions

10 Il s'agit de Madison, de David Riesman, d'Arthur Bentley, de David Truman, de Robert Dahl et de tant d'autres auteurs de la science politique américaine qui considèrent que le pluralisme politique est le trait essentiel du système politique américain. Pour ces politologues, le pluralisme politique implique une distribution du pouvoir dans la société et la possibilité pour les groupes (associations civiles et partis politiques) de négocier dans un mouvement permanent de marchandage légitime. Il y a principalement deux types de théories pluralistes : le pluralisme classique, plus connu sous le nom de « group politics » et le néo-pluralisme ou pluralisme critique, renvoyant au néocorporatisme (Schmitter).

11 Voir: Michel OFFERLE, Sociologie des groupes d'intérêt, Paris, LGDJ / Montchrestien, $1998^{2}$. 
est rendue légitime par la multiplicité des intérêts sociaux et la compétition pour le pouvoir. C'est là, semble t-il, l'héritage de Madison dans la théorie démocratique. C'est dans le Fédéraliste ${ }^{12}$ que l'on trouve une première défense de ce pluralisme, car, comme l'écrit Madison,

« étendez sa sphère, elle comprendra une plus grande variété de partis et d'intérêts, vous aurez moins à craindre de voir une majorité avoir un motif commun pour violer les droits des autres citoyens. ».

Pour cet auteur, le pluralisme est un moyen qui favorise la conservation de la sécurité, de l'égalité, de la liberté et l'auto-gouvernement du peuple. Il empêche l'apparition et l'installation du pouvoir absolu, unique ou totalitaire d'une majorité. Il ne suffit pas, selon Madison, d'avoir la majorité électorale pour prétendre à la légitimité démocratique.

À la suite de Madison, Tocqueville, dans La Démocratie en Amérique, fait de la défense du pluralisme le moteur de la démocratie. Le pluralisme permet d'éviter les excès de l'individualisme en favorisant le développement quasi illimité de la vie associative. Comme Madison, les pluralistes américains, de David Riesman à Robert Dahl, pensent donc que le pluralisme des factions, des partis et des intérêts est source de stabilité et l'expression la plus appropriée de la démocratie :

« Like Madison, they accepted that a fundamental purpose of government is to protect the freedom of factions to further their political interests while preventing any individual faction from undermining the freedom of others. Unlike Madison, however, pluralists argued (...) that far from posing a major threat to democratic associations, factions are a structural source of stability and the central expression of democracy. For pluralists, the exis-

12 - MADISON, « Federalist $10 »$, in A. HAMILTON, J. MADISON, J. JAY, The Federalist Papers (1787), ed C. Rossiter, New American Library, 1961 ; trad. fr. : Le Fédéraliste, Paris, L.G.D.J., 1957. Lire la présentation succincte et globale de : Laurent BOUVET et Thierry CHOPIN, Le « fédéraliste » : la démocratie apprivoisée, Paris, Michalon, 1997. 
tence of diverse competitive interests is the basis of democratic equilibrium and of favourable development of public policy. $» 13$.

Ce qui préoccupe en effet les pluralistes, c'est non pas l'exigence utilitariste de maximisation des intérêts propres, la tyrannie de la satisfaction du désir d'un individu, d'une minorité voire d'une majorité, mais de veiller à ce que le pouvoir soit partagé et soumis à la compétition du plus grand nombre de groupes représentant divers intérêts. Voilà l'objet du célèbre livre de Robert Dahl sur le pouvoir démocratique aux États-Unis ${ }^{14}$.

Le politologue américain estime que le pouvoir, pour être démocratique, doit être soumis à l'épreuve de la compétition pluraliste, en mettant aux prises une multiplicité de groupes qui cherchent à influencer les politiques publiques (public policies). Une démocratie véritable se reconnait, selon Dahl, à deux critères : l'existence d'une participation effective et l'organisation régulière d'une compétition réelle. Il pense que s'il existe des systèmes électoraux compétitifs, mettant en concurrence une multiplicité d'individus ou de partis, alors seront garanties les libertés démocratiques. La ligne de démarcation entre le régime autoritaire et le régime démocratique passe par le pluralisme, par le biais d'élections régulières et d'une compétition politique entre les individus, les groupes et les partis. Un véritable démocrate doit être un pluraliste convaincu :

«To destroy pluralism, écrit Dahl, would require an authoritarian regime that would devote extraordinary coercion to that end. A monistic system is an ideal appropriate to authoritarianism ; it cannot be an ideal for democrats. For better or worse, a modern democrat must also be a pluralist. » 15 .

13 David HELD, Models of Democracy, Cambridge, Polity Press, 1987, pp. 187188.

14 Robert A. DAHL, Who governs ? Trad. en français : Qui gouverne, Paris, A. Colin, 1971.

15 Robert A. DAHL, Democracy and its critics, New Haven and London, Yale University Press, 1989, p. 298. 
Ainsi donc, le caractère démocratique d'un régime politique repose sur l'existence d'une pluralité sociale et politique. Si tous les citoyens n'ont pas accès au pouvoir, au minimum ils doivent pouvoir exercer un contrôle sur les gouvernants par le biais d'élections régulières. Les élections sont un instrument du pluralisme en tant qu'elles permettent à différents groupes en compétition d'exprimer leurs prétentions. Elles le sont aussi en tant qu'elles permettent à des citoyens ordinaires de choisir leurs dirigeants.

À la suite de Madison, Dahl défend l'idée que le pluralisme ou l'existence d'une véritable diversité d'intérêts et de perspectives, protège la société de la «tyrannie d'une majorité factionnelle» (« the tyranny of a factitious majority »). Dans une démocratie pluraliste, le pouvoir doit être effectivement partagé ou du moins il ne saurait être entre les mains d'un seul individu ou groupe. Dans une société démocratique, l'exercice d'un pouvoir se mesure à la capacité d'utiliser des ressources pour avoir une influence dans l'élaboration et la prise des décisions. Les ressources sont diverses. Diverses aussi sont les capacités d'accéder à tous les types de ressources. Il y a beaucoup d'inégalités sociales (sur le plan de la santé, de la richesse, de l'instruction...).

Cependant, comme différents groupes ont accès à différentes sortes de ressources, il y a une multiplicité d'influences possibles. Dans cette perspective, le pouvoir ne saurait être concentré en un centre unique. Le pouvoir est dispersé puisqu'il y a une «pluralité de pôles de pression » (" plurality of pressure points »). Selon la théorie classique du pluralisme, il n'y a pas de pouvoir solitaire, mais une diversité de centres d'élaboration de politiques publiques concurrentielles et de prise de décisions («a variety of competing policy-formulating and decision-making centres »). C'est cela que Dahl appelle le régime polyarchique. David Held résume cette conception pluraliste du pouvoir en écrivant : 
«In the pluralist account, power is non-hierarchically and competitively arranged. It is an inextricable part of an " endless process of bargaining " between numerous groups representing different interests, including, for example, business organizations, trade unions, political parties, ethnic groups, students, prison officers, women's institutes, religious groups. $\gg 16$.

Selon cette vision dahlienne de la démocratie pluraliste ou du pluralisme démocratique, les termes de «pluralisme » et "pluraliste » renvoient à l'existence d'une pluralité d'organisations relativement autonomes et indépendantes, dans la sphère étatique. Il s'agit donc d'un pluralisme organisé, institutionnalisé (« organizational pluralism ») dont le pluralisme des partis politiques. Cinq (5) critères définissent cet idéal démocratique :

1- l'égalité du vote (« equality in voting »)

2- la participation citoyenne (« effective participation »)

3- la clairvoyance politique (« enlightened understanding »)

4- le principe du contrôle (« final control over the agenda »)

5- le principe d'inclusion (« inclusion »)

Nous le voyons donc: Dahl place les partis politiques au cœur du pluralisme démocratique. Ceux-ci permettent la conquête du pouvoir par le biais des élections et protègent la démocratie contre la dérive monarchique. Pour consolider ce trait distinctif de la démocratie, le pluralisme est une procédure d'institutionnalisation de la compétition politique et de choix des élites partisanes. Telle est le conception développée par Schumpeter.

Pour Joseph SCHUMPETER, dans Capitalisme, Socialisme et Démocratie * le pluralisme politique désigne la multiplicité des groupes, notamment des partis politiques, qui sont engagés dans une compétition électorale pour conquérir le pouvoir politique. Pour lui, la

16 David HELD, Models of Democracy, op.cit., p.189.

* Texte disponible dans Les Classiques des sciences sociales. JMT. 
démocratie est une méthode de gouvernement qui repose sur l'idée qu'il n'y a aucune doctrine générale qui s'imposerait à tous, mais elle permet à la société de s'auto - administrer, de s'autoréguler par la libre compétition des groupes partageant les charges et les privilèges de la coopération sociale. La théorie de Schumpeter taxe d'irréaliste l'idéal de la démocratie athénienne. Se voulant réaliste, Schumpeter affirme que ce qui distingue les démocraties des non démocraties ce sont les méthodes de sélection des leaders politiques ${ }^{17}$.

Du reste, la démocratie ne requiert pas une intense participation de tous les citoyens ; elle peut très bien fonctionner sans cela. Schumpeter s'accorderait bien avec Lipset qui a écrit que «l'apathie politique pourrait refléter la santé d'une démocratie ». Il est empiriquement établi que l'électorat est plus apathique et moins informé que les théoriciens de la démocratie ne l'admettent généralement ou que les citoyens, individuellement, ont peu d'impact direct sur les processus politiques. En réalité, les vrais décideurs et faiseurs d'opinion («opi-

17 Dans sa définition classique, Joseph A. SCHUMPETER considère que la démocratie est un ensemble de dispositifs procéduraux ou de méthodes électorales de choix des élites politiques : «le système institutionnel, aboutissant à des décisions politiques, dans lequel des individus acquièrent le pouvoir de statuer sur ces décisions à l'issue d'une lutte concurrentielle portant sur les votes du peuple »: J. A. SCHUMPETER, Capitalisme, socialisme et démocratie, Paris, Payot, 1951, pp. 329-330, c'est nous qui soulignons. Cette conception schumpetérienne de la démocratie a été qualifiée d'élitiste. Selon elle en effet, ce ne sont pas les électeurs qui déterminent la politique dans une démocratie représentative. Les représentants ne sont pas contraints de mettre en oeuvre une volonté populaire exprimée lors des élections. Le dispositif démocratique laisse une marge d'indépendance des gouvernants à l'égard des électeurs. Le vote est une épreuve concurrentielle et non le choix d'une politique ; il désigne seulement, parmi plusieurs adversaires, ceux qui sont chargés de décider. Le vote démocratique consacre le pluralisme compétitif et le choix des élites politiques. La magistrale étude de Bernard MANIN, remarquable par son érudition précise et son argumentation, présente le rapport entre la prise des décisions publiques (versant élitiste) et les volontés de l'électorat (versant populaire) comme principe de la démocratie représentative: Bernard MANIN, Principes du gouvernement représentatif, Paris, Flammarion, 1996, pp. $171-214$. 
nion-makers »), ce sont les représentants, les élites. Aux yeux de Schumpeter, le citoyen est isolé, faible et vulnérable dans un univers politique marqué par «le choc compétitif des élites» (« the competitive clash of elites $»)$.

Cette conception élitiste de la démocratie, que Schumpeter partage avec Weber, se veut « réaliste ». Il s'agit, en effet, de définir la démocratie en se focalisant sur les pratiques et les institutions de ce que l'on appelle conventionnellement la «démocratie» en Occident. L'étude de la nature empirique et descriptive de la démocratie porte aussi sur les mécanismes à travers lesquels les citoyens peuvent contrôler les dirigeants politiques (élections régulières, groupes de pression).

La question demeure de savoir ce que vaut la problématique du pluralisme devant cette insistance sur l'élitisme (« elite politics»). Ce qui est sûr, c'est que pour Schumpeter le pluralisme n'est légitime que si le système permet la compétition électorale. Le pluralisme est une exigence méthodique de la démocratie telle que la conçoit Schumpeter, c'est-à-dire un lieu de compétition entre les élites. Mais, cette notion de pluralisme n'est pas arithmétique. Elle ne se réduit pas à une simple multiplication numérique des organisations sociales, économiques et politiques.

Néanmoins, l'insistance sur la nature « réaliste » et " élitiste » de la démocratie pluraliste rend la théorie de Schumpeter partiale et incomplète, car elle a tendance à négliger la portée réelle de la participation citoyenne. Par exemple, elle néglige la dynamique politique des groupes intermédiaires, tels que les associations communautaires, religieuses, syndicales, les organisations économiques par lesquels les citoyens ordinaires sont reliés, de différentes manières, à une variété de types d'institutions et de pratiques. Par ces canaux de la société civile s'exprime aussi le pluralisme démocratique. De plus, en concevant la démocratie d'une façon exclusivement «empirique », Schumpeter manque d'examiner les questions relatives à la justification, à la régu- 
lation, aux caractéristiques et conditions générales du pluralisme démocratique. David Held critique le réalisme webérien et schumpetérien en écrivant :

« Their « realism» entailed conceiving of democracy in terms of the actual features of Western polities. In thinking of democracy in this way, they recast its meaning and, in so doing, surrendered the rich history of the idea of democracy to the extent. Questions about the appropriate extent of citizen participation, the proper scope of political rule and the most suitable spheres of democratic regulation - questions that have been part of democratic theory from Athens to nineteenth-century England - are put aside, or, rather, answered merely by reference to current practice. The ideals and methods of democracy become, by default, the ideals and methods of the existing democratic system. Since the critical criterion adjudicating between theories of democracy is their degree of « realism ", models which depart from, or are in tension with, current democratic practice can be dismissed as empirically inaccurate, « unreal», and undesirable. $\gg 18$

La logique du multipartisme 19 n'est pas d'éparpiller les énergies et les initiatives politiques, mais de les focaliser autour de la riche complexité sociale pour construire l'unité dans la diversité. Sans doute, la métaphore qui en rend compte le mieux est celle du faisceau: «E pluribus unum ». Le pluralisme des perspectives politiques désigne en clair la fin du monolithisme, les possibilités de rupture et de mobilité dans le champ politique. Toutefois, si affirmé et solide que soit le pluralisme, il est raisonnable de chercher à ramener la pluralité des sphères à la nécessaire unité d'un vivre ensemble. Le pluralisme n'est pas anarchie ; l'unité et la pluralité s'appuient l'une sur l'autre comme deux exigences dialectiques dans une réciproque limitation.

Il ne saurait être question de limiter le pluralisme politique à une arithmétique ou à une multiplication des partis politiques ou des groupes d'intérêts. Il ne faut pas confondre la simple fragmentation du sys-

18 David HELD, Models of Democracy, op.cit., pp. 196-197.

19 Cf. Manfred J. HOLLER (ed), The Logic of Multiparty Systems, Dordrecht, Boston, Lancaster : Kluwer Academic Publishers, 1987, 446p. 
tème des partis («mere fractionalization ») ou le nombre des partis et le pluralisme politique, que Sartori qualifie de «pluralisme polarisé » ( polarized pluralism) ${ }^{20}$. Selon Sartori, le pluralisme polarisé est caractérisé par huit (8) propriétés essentielles :

1) la présence de partis opposés au système (anti-system parties );

2) l'occupation du « centre métrique » (the « metrical center») par un parti ;

3) l'existence d'oppositions bilatérales (« bilateral oppositions »);

4) la polarisation, c'est-à-dire la distance idéologique entre les partis dans un spectre (« ideological distance between parties on a spectrum $\gg)$;

5) tendances de vote centrifuge (centrifugal voting tendencies);

6) la structuration idéologique (ideological patterning), avec les partis politiques orientant les opinions des citoyens vers la compétition ;

7) Des oppositions irresponsables («irresponsible oppositions »), où certains partis sont en permanence exclus du gouvernement tandis que d'autres gouvernent presque toujours ;

8) une politique de "surenchère ", avec les partis qui multiplient les promesses à la recherche d'un soutien.

Quelles sont les implications d'un tel pluralisme politique au regard de la nécessaire stabilité politique 21 ? Selon une thèse tradition-

20 Cf. G.SARTORI, « European Political Parties ; The case of Polarized Pluralism », in J. La Palombara and M. Werner (eds), Political Parties and Development, Princeton: Princeton University Press, 1966, pp. 137-176 ; Parties and Party Systems : A framework for Analysis, New York: Cambridge University Press, 1976, pp.132-144 ; G.SANI and G. SARTORI, «Polarization, Fragmentation and Competition in Western Democracies », in H. Daalder and P. Mair (eds), Western European Party Systems, London: Sage, 1983, pp. 307-340. Voir aussi G.Bingham Powell, Jr., « The competitive consequences of polarized pluralism », in Manfred J. HOLLER (ed), The Logic of Multiparty Systems, Dordrecht, Boston, Lancaster : Kluwer Academic Publishers, 1987.

21 Voir : G.B.Jr, POWELL, Contemporary Democracies : Participation, Stability and Violence, Cambridge : Cambridge University Press, 1982 ; L. HUR- 
nelle, le pluralisme des partis est considéré comme résultat de la segmentation sociale et conduit, de ce fait, à des gouvernements faibles et instables. On peut opposer à cette conception péjorative que le multipartisme n'est pas de soi une force d'inertie ou un facteur d'instabilité, «which translates social heterogeneity and immobilism into political instability ». On peut considérer, avec Arendt Lijphart, que le multipartisme peut très bien favoriser la stabilité politique si d'autres institutions politiques de type consociatif (consociational devices) sont mises en place ${ }^{22}$. Le multipartisme peut aussi être combiné à une variété de modèles de coalition gouvernementale. Le multipartisme ne peut être réduit à un instrument d'instabilité. Il doit plutôt être conçu comme un moyen d'exercice de la citoyenneté, d'expression de la pluralité et des préférences. La logique du multipartisme est celle de la représentation de la pluralité des choix et des préférences politiques :

« Multipartism cannot be approached as the sole cause of political instability, merely mirroring extensive cleavages. Such a model would seriously neglect the institutional context of the party system as an entity in the overall political system. Theoretically, multipartism may be identified as an instrument for the exercise of citizens, influence on government policymaking. By voting for a variety of parties citizens express their different preferences about policies which reflect the basic connection between multipartism and representation. Multipartism enters into a system of institutions for collective choice. $\gg 23$

WITZ, « Democratic Political Stability : some traditional hypotheses reexamined », in Comparative Political Studies, 4, 1972, pp.476-490.

22 Arendt LIJPHART, The politics of accomodation. Pluralism and democracy in the Netherlands, Berkeley, University of California Press, 1968; L. SYLLA, La gestion démocratique du pluralisme socio-politique en Afrique. Deux modèles : démocratie concurrentielle et démocratie consociationnelle, Association internationale de science politique, Comité de recherche sur le pluralisme socio-politique, Colloque sur le gouvernement des sociétés plurales d'Afrique, Rome, août-sept. 1981.

23 G.B. Jr POWELL, «The competitive consequences of polarized pluralism », in Manfred J. HOLLER (ed), The Logic of Multiparty Systems, Dordrecht, Boston, Lancaster : Kluwer Academic Publishers, 1987, pp.156-157. 
Ainsi, il nous apparaît que le véritable enjeu du pluralisme partisan n'est pas d'éparpiller les forces politiques en des directions incohérentes et d'installer l'instabilité permanente. Dans une démocratie pluraliste, le système de partis structure les institutions, des relations et des pratiques à travers lesquelles les citoyens expriment leurs choix collectifs. En d'autres termes, le multipartisme est, grâce au vote, ce qui rend possible la représentation des intérêts multiples et l'expression citoyenne d'une volonté politique commune. C'est dire que le problème du pluralisme réside dans la dialectique de la multiplicité et de l'unité. Il convient, pour bien approfondir le sens de ce propos, de faire une sorte généalogie conceptuelle (philosophique) du pluralisme politique. 


\section{2.- Philosophie du pluralisme : entre l'un et le multiple.}

$\underline{\text { Retour à la table des matières }}$

Il semble que le terme de "pluralisme » a été utilisé pour la première fois en 1720 par le philosophe allemand Christian Wolff. Mais, ce n'est certainement pas lui le concepteur du discours philosophique du pluralisme 24 . Pour reconstituer la généalogie conceptuelle du pluralisme, il faudrait, sans doute, remonter jusqu'à la philosophie grecque naissante.

24 Le Larousse du $X X^{e}$ siècle définit le pluralisme comme une «doctrine philosophique qui proscrit la recherche de l'unité et des lois universelles, parce que dans ce monde, il n'y a que des êtres multiples, individuels ». Le Vocabulaire technique et critique de la philosophie d'André Lalande le définit comme une "doctrine selon laquelle les êtres qui composent le monde sont multiples, individuels, indépendants, et ne doivent pas être considérés comme de simple modes ou phénomènes d'une réalité unique et absolue ». En cela, la philosophie du pluralisme s'oppose au monisme et soutient la thèse selon laquelle l'altérité, la diversité, l'hétérogénéité, la discontinuité l'emportent partout, dans la vie comme dans l'ordre scientifique, sur l'identité, l'homogénéité et la continuité. La philosophie moderne du pluralisme s'enracine doublement dans la considération du "polythéisme des valeurs » (Nietzsche, Max Weber) et dans une nouvelle conception de l'individualité ou de la subjectivité, de la raison et de l'histoire. Voir sur cette question: Alain RENAUT, L'ère de l'individu : contribution à une histoire de la subjectivité, Paris, Gallimard, 1989 ; L'individu : remarques sur la philosophie du sujet; Sylvie MESURE et Alain RENAUT, La guerre des dieux. Essai sur la querelle des valeurs, Paris, Grasset, 1996. Le sujet ne se définit plus par un arrimage à un monde clos de significations, mais par référence à lui-même (Descartes), mais aussi, de plus en plus, dans son rapport aux valeurs multiples d'un monde désenchanté. Les morales se démultiplient, la raison totalitaire, unique détentrice de la vérité, s'affaiblit au profit d'une raison communicationnelle et argumentative (Habermas). Alors, s'imposent progressivement les philosophies de la pluralité, de l'altérité et de la dialectique contre les systèmes de l'unité et de l'identité. Voir : William JAMES, A pluralistic Universe, London, Longmans, Green \& Co., 1909 ; Jean WAHL, Les philosophies pluralistes d'Angleterre et d'Amérique, Paris, Alcan, 1920. 
Pour les premiers philosophes grecs, appelés « Présocratiques » 25, penser la pluralité, c'est s'interroger, à la suite d'Anaxagore de Clazomènes et d'Héraclite d'Ephèse, sur les relations entre l'Un et la Multiplicité. Le pluralisme consiste, pour l'essentiel, à chercher à expliquer la totalité ou un système à partir de ses "parties dispersées, fragmentées, discontinues et irréductibles » 26.

Ainsi, la vision de la pluralité ne s'oppose pas stricto sensu à l'unité. La conscience de la diversité et l'exigence d'unité s'interpénètrent, car le monde est une « réunion de parties » disparates au sein d'un tout composite. Le pluralisme est le principe de l'unité dans la diversité. Il est l'envers de toute réclusion dans un idéal totalitaire, moniste. Il n'est pas un agrégat accidentel d'idiosyncrasies particulières ou de traditions et d'organisations autarciques, indifférentes les unes aux autres et qui seraient contraintes de s'intégrer au sein d'un système global formel. Une société pluraliste est une société ouverte ${ }^{27}$, traversée par différentes sphères de pouvoirs et caractérisée par l'existence d'allégeances multiples.

«Une société pluraliste se présente d'une manière générale comme un ensemble composé (à compartiments) librement aggloméré. Les différentes sphères ou secteurs qui la constituent ont gardé une bonne part de leur autonomie originelle. Il s'agit d'autant de domaines irréductibles, en interaction permanente $» 28$.

25 Voir : Les penseurs grecs avant Socrate. De Thalès de Milet à Prodicos, traduction, préface et notes par Jean Volquin, Paris, Garnier-Flammarion, 1964.

26 André REZLER, Le pluralisme : aspects théoriques et historiques des sociétés ouvertes.- Genève / I.U.E. : Georg Eshel, 1990,, p.11.

27 Cette notion de "société ouverte " se trouvait déjà chez Henri BERGSON dans Les deux sources de la morale et de la religion, Paris, P.U.F., 1932, mais elle a été généralisée par Karl POPPER dans son livre La société ouverte et ses ennemis (en anglais: The Open society and Its Enemies). Les sociétés ouvertes et pluralistes sont des formes d'organisation sociale nées de la décomposition des sociétés fermées et tribales. Celles-ci sont caractérisées par la foi dans les tabous magiques, tandis que celles-là désignent des groupes d'hommes ayant une attitude critique à l'égard de toute croyance et fondant leurs actions sur l'autorité de leur propre intelligence.

28 André REZLER, Le pluralisme, op. cit., p. 56. Mis en italique par l'auteur. 
S'inspirant de cette conception, la philosophie politique contemporaine envisage le pluralisme, dans sa dimension sociale et politique, comme une dialectique entre le consensus et le dissensus. C'est dans cette perspective qu'il me semble opportun d'examiner la conception du pluralisme démocratique chez John RAWLS, par exemple. Il ne saurait être question ici d'analyser la théorie rawlsienne de la « justice comme équité $\gg{ }^{29}$. Qu'il nous suffise ici de résumer sa conception du pluralisme politique, notamment l'articulation chez lui entre pluralisme et consensus.

Tout d'abord, Rawls distingue le «pluralisme simple » et le «pluralisme raisonnable $»$. Il tient pour acquis le $«$ fait du pluralisme $»$, c'est-à-dire l'existence d'une pluralité de doctrines compréhensives, morales, politiques, philosophiques et religieuses. Cette situation n'est pas une circonstance ou « une simple contingence historique, (mais) un trait permanent de la culture publique des démocraties » ${ }^{30}$, signifiant la liberté des individus de choisir leur système de valeurs et l'égalité de ces systèmes entre eux, puisque aucun d'entre eux ne peut

29 Cf. John RAWLS, Théorie de la Justice. Trad. de l'anglais par Catherine Audard, Paris, Seuil, 1987. Pour des analyses, on pourra consulter : Brian BARRY, The Liberal Theory of justice. A critical examination of principal doctrines in a "A Theory of justice" by John Rawls, Oxford : Oxford University Press, 1973 ; H. G. BLOCKER, E. H. SMITH, John Rawl's Theory of social justice. An Introduction, Berkeley and Los Angeles : University of California Press, 1982 ; Jean LADRIERE et Philippe VAN PARIJS (dirs), Fondements d'une théorie de la justice. Essais critiques sur la philosophie politique de John Rawls, Louvain-la-Neuve, Peeters ; Paris, Vrin, 1984 ; Otfried HOFFE, L'État et la justice. Les problèmes éthiques et politiques dans la philosophie anglo-saxonne, John Rawls et Robert Nozick, Paris: Vrin, 1988 ; N. DANIELS (ed.), Reading Rawls, Standford: Standford University Press ; René SEVE et alii, Individu et justice sociale. Autour de John Rawls, Paris : Seuil, 1988 ; Ph. Van Parijs, Qu'est-ce qu'une société juste ? Introduction à la pratique de la philosophie politique, Paris : Seuil, 1991 ; Jean - Pierre DUPUY, Le sacrifice et l'envie, Paris : Seuil, 1992.), 1993.

30 John RAWLS, Justice et démocratie. Introduction, présentation et glossaire par Catherine Audard. Trad. de l'anglais par C. Audard, P. De Lara, F. Piron et A. Tchoudnowsky, Paris, Seuil, 1993, p.325. 
et ne doit s'imposer à tous, à moins de recourir à la violence, à la persécution. Le problème est de contrôler la pluralité des doctrines ("pluralisme simple ») de manière à ne pas détruire l'unité et la stabilité de la société. Si la reconnaissance du pluralisme exclut l'idéal d'une société politique homogène, unanimiste et totalitaire, elle n'implique pas non plus la dispersion anarchique.

Rawls prône un "pluralisme raisonnable », caractérisé par l'existence de doctrines certes incompatibles entre elles, mais raisonnables. Le pluralisme raisonnable est « le résultat normal de l'exercice de la raison humaine dans le cadre des institutions libres d'un régime démocratique constitutionnel $\gg{ }^{31}$. La notion de pluralisme raisonnable renvoie à l'exercice de la raison raisonnable, c'est-à-dire à la capacité de s'abstraire des intérêts immédiats et bornés pour envisager les situations selon des principes politiques universalisables (Kant) et justifiables. Pour ce faire, le pluralisme doit être appuyé sur des « jugements bien pesés ", c'est-à-dire des critères de justification morale.

Rawls propose une "morale concrète » raisonnable et non une doctrine générale exhaustive. L'entreprise philosophique de Rawls consiste dans « la défense d'une croyance raisonnable en la possibilité réelle d'un régime constitutionnel juste $»{ }^{32}$. Pour le philosophe libéral américain, le fait du pluralisme, caractéristique de la modernité démocratique, n'exclut pas la recherche d'un consensus.

On ne comprendrait pas la portée démocratique et libérale de la théorie de la justice comme équité sans ce couple indissociable pluralisme / consensus ${ }^{33}$. Ce binôme est à la fois l'acte de référence (« de quoi parle t-on? ») et l'acte de prédication (« qu'en dit-on ?) du sens

31 John RAWLS, Libéralisme politique. Trad. de l'américain par Catherine Audard, Paris, P.U.F., 1995, p.4.

32 J. RAWLS, «L'idée d'un consensus par recoupement », in Justice et démocratie, op.cit., p.283.

33 Voir : Catherine AUDARD, «Pluralisme et consensus : une philosophie pour la démocratie ?», Critique, n 505-506, juin-juillet 1989. 
de la justice politique. Sans l'existence (jugement ontologique) du pluralisme, le consensus devient tyrannique, unanimisme étouffant qui embrigade l'expression des libertés individuelles (jugement axiologique). On ne doit accepter le consensus comme norme politique que sous condition que le pluralisme raisonnable soit sauvegardé. Dans une société pluraliste, l'unité est le résultat d'un «consensus par recoupement » (« overlapping consensus ») ${ }^{34}$.

Le pluralisme est fondé sur une égalité universelle des droits civiques ; il vaut pour toute communauté humaine, pour toute communauté d'êtres raisonnables. L'universalisme du pluralisme est un principe négatif, qui définit ce sans quoi il n'y a pas d'humanité possible : « La société est plusieurs », selon Georges Balandier.

Cette conception universaliste est au cœur de la philosophie politique de Michaël Walzer ${ }^{35}$, qui partage avec Isaiah Berlin une option résolue en faveur du pluralisme ${ }^{36}$. Walzer se situe bien dans une tradition de pensée, l'universalisme, qui va de Kant à Habermas, en incluant Rawls et Dworkin ${ }^{37}$. Mais le philosophe américain ne suit pas jusqu'au bout cette perspective universaliste 38 à laquelle il reproche

34 J. RAWLS, «Le domaine du politique et le consensus par recoupement », in Justice et démocratie, op.cit., pp.323-356.

35 Voir : Michaël WALZER, Sphères de justice. Une défense du pluralisme et de l'égalité, trad. fr. Pascal Engel, Paris : Seuil, 1997 ; Pluralisme et démocratie, trad. collective, Introduction de Joël Roman, Paris : Editions Esprit, 1997.

36 Voir: Michaël WALZER, Pluralisme et démocratie, op. cit., pp. 131-147 (« Comment valoriser le pluralisme ? Une lecture d'Isaiah Berlin ».) ; pp. 207220 (« Eloge du pluralisme démocratique. Entretien avec Michaël Walzer »).

37 Voir : Ronald DWORKIN, Taking rights seriously, Cambridge, Mass., Harvard University Press, 1978.

38 M. WALZER, Pluralisme et démocratie, op. cit., pp. 83-109 (« Les deux universalismes »). Le philosophe américain oppose : 1)- un « universalisme de surplomb », qui « considère qu'il n'y a qu'un seul Dieu, donc une seule loi, une seule justice, une seule conception de la vie bonne ou de la société bonne ou du bon régime, un salut, un messie, un millenium pour toute l'humanité » (p. 84). Il est produit par les religions messianiques. 2)-un « universalisme réitératif $»$, dont l'exemple est donné par la sortie d'Egypte, l'exode du peuple 
son caractère abstrait, constructiviste et la mise entre parenthèses de tout élément empirique. C'est pourquoi, Walzer, se rapprochant des auteurs dits communautariens comme Alasdair MCINTYRE 39 ou Michaël SANDEL ${ }^{40}$, fait droit au particularisme des communautés politiques et des traditions vécues. Fort de cette position médiane entre libertariens et communautariens ${ }^{41}$, Walzer propose une philosophie qui fragmente l'aspiration universelle de justice en autant de figures d'organisation sociale. Cette philosophie du pluralisme est à la fois universaliste et particulariste :

«C'est reconnaître l'existence de la différence - partout. La reconnaissance est universelle, tandis que ce qui est reconnu est local et particulier. Cela pourrait être envisagé comme un universalisme réitératif : la création des biens et des identités a lieu constamment et jamais de la même manière $\gg 42$.

Le pluralisme s'appréhende de l'intérieur de chaque communauté historique et se comprend comme obligation de séparer les différentes « sphères de justice ». Il ne s'impose pas d'en haut ou verticalement, mais procède horizontalement du désir singulier d'autonomie de chaque individu, de chaque groupe. Il se configure dans les divers cercles d'appartenance, impliquant ainsi la distinction des ordres de distribu-

juif. Il n'y a un modèle universel de l'exode, mais une expérience singulière de la libération, qui peut être répétée à nouveaux frais pour chaque peuple. Ce n'est pas le fait historique qui est universel, il est singulier, mais il a signification universelle.

39 Voir : Alasdair MACINTYRE, After Virtue, Notre Dame, Ind., University of Notre Dame Press, 1981 ; Whose Justice ? Which Rationality ?, London, Duckworth, 1988. Trad. fr. Quelle justice ? Quelle rationalité ?, Paris, P.U.F., 1993.

40 Voir : Michaël SANDEL, Liberalism and its critics, New York, New York University Press, 1984.

41 Voir : Daniel BELL, Communitarianism and its critics, Oxford, Clarendon Press, 1993.

42 Michaël WALZER, Pluralisme et démocratie, op. cit., p. 214. 
tion des biens matériels et symboliques ${ }^{43}$, des normes juridiques et morales.

« Je recours à la notion de pluralisme dans deux sens (...). Il y a d'abord un pluralisme qui se réfère aux biens sociaux et aux "sphères de justice" que ceux-ci constituent, avec leurs différents principes de distribution et les procédures qui leur correspondent. Il y a ensuite le pluralisme des identités sociales et des cultures ethniques et religieuses à partir desquelles elles sont engendrées $\gg{ }^{44}$.

Cette perspective walzérienne invite à une règle de prudence ${ }^{45}$, qui consiste à se rapporter non pas à un seul système de valeurs, mais à plusieurs, mettant ainsi fin à la "prédominance et au monopole » d'une sphère donnée. Le pluralisme ou la différence des sphères et des principes de justice s'appuie sur ce que Walzer nomme le « régime de l'égalité complexe » ou la complexité sociale. La démocratie libérale est la forme d'organisation politique qui garantit le mieux le principe pluraliste, en permettant le libre débat et l'exercice de la citoyenneté électorale.

Nous avons là une nouvelle interprétation du libéralisme moderne. Pour Walzer, l'originalité de cette idéologie ne réside pas d'abord dans l'individualisme, mais dans l'autonomie des diverses sphères

43 Voir Paul RICOEUR, Soi-même comme un autre, Paris, Seuil, 1990, p. 293 et ss.

44 Michaël WALZER, Pluralisme et démocratie, op. cit., p. 212.

45 Voir: Gil DELANNOI, Eloge de la prudence, Paris, Berg, 1993 ; Guy LAFOREST, De la prudence, Québec, Boréal, 1993. Jean LECA ( dans « La démocratie à l'épreuve des pluralismes ", Revue Française de science politique (46), 2, avril 1996, p. ) écrit : «Le pluralisme n'est pas un système et ses rapports avec la démocratie ne peuvent être réglés par un diktat philosophique ou sociologique mais par une analyse contextuelle et une démarche prudentielle tenant compte des différents héritages historiques». M'inspirant de cette règle de prudence, je m'évertuerai à ne pas substantialiser le concept de pluralisme. 
d'existence ou la séparation des institutions religieuses, politiques, économiques ${ }^{46}$.

« La véritable liberté, explique Joël Roman, ne consiste pas à pouvoir choisir en toute autonomie individuelle, mais bien à se voir garantir cette autonomie individuelle par l'autonomie qui sépare et divise les institutions. (...). Il s'agit donc d'inverser le chemin de Rawls : partir au contraire de l'exigence de justice telle qu'on doit pouvoir la manifester en mettant en évidence la nécessité de séparer les institutions pour arriver à l'individu, beaucoup plus que de partir des individus pour arriver à une règle de justice universelle $\gg 47$.

On peut estimer que Walzer est à mi-chemin du libéralisme et du communautarisme ${ }^{48}$. Il soutient que la liberté de l'individu réside dans la multiplicité des sphères d'allégeance, qu'elle se vit à travers des identités mobiles et qu'il serait donc dangereux et illusoire de la réduire en une identité figée. Mais, une telle perspective de la multiappartenance ne signifie pas la dissolution de toute identité. Le pluralisme n'est pas une négation de toute forme d'appartenance (membership) : l'individu libre s'appartient à lui-même (pluralisme des individus) et appartient à une communauté politique (pluralisme des groupes), partageant un certain nombre de valeurs communes (nation, nationalité), dans les limites d'un territoire déterminé.

Au total, il apparaît que la philosophie du pluralisme politique renvoie à la problématique de la social-démocratie libérale et pluraliste et permet de repenser les idéaux de la " gauche » ${ }^{49}$, c'est-à-dire les valeurs de liberté, d'égalité et de solidarité.

46 Voir : Michaël WALZER, Pluralisme et démocratie, op. cit., pp. 29-51 (« La justice des institutions »).

47 Joël ROMAN, «Le pluralisme de Michaël Walzer» (Introduction), in M. Walzer, Pluralisme et démocratie, op. cit. , p. 23.

48 Voir : M. WALZER, Pluralisme et démocratie, op. cit., pp. 52-82 (« La critique communautarienne du libéralisme »).

49 Voir : M. WALZER, Pluralisme et démocratie, op. cit., pp. 149-165. (« Pluralisme et social-démocratie »). 
«Le pluralisme qu'implique la liberté est celui des traditions ethniques, culturelles et religieuses et des groupes d'hommes et de femmes qui les soutiennent. (...). Le pluralisme qu'implique l'égalité est celui des biens sociaux différents et des sphères autonomes (...). Le pluralisme qu'implique la solidarité est constitué par l'ensemble des groupes et associations où les gens se rassemblent pour soutenir un mode de vie, encourager une conception de la justice ou défendre un ensemble d'intérêts » 50 .

La méthode de la prudence requiert d'envisager une critique théorique de la signification du pluralisme au regard de la spécificité du politique. A cet égard, il serait utile ici d'évoquer la réflexion qu'avait formulée dans les années 30, bien avant Rawls et Walzer, le juriste allemand, Carl Schmitt. Cette réflexion garde encore son actualité devant les ambiguïtés politiques du pluralisme ${ }^{51}$.

Carl Schmitt ne peut déconnecter la conception de la démocratie et du pluralisme du souci de la vérité absolue du souverain et de la préoccupation de l'unité politique. De même qu'il est illusoire de se donner à soi-même ses propres fondements, de même la démocratie ne saurait se contenter d'un pluralisme relativiste. Certes, Schmitt accepte le « fait du pluralisme » ${ }^{52}$. Il sait que la spécificité de la démocratie

50 M. WALZER, Idem, p. 160.

51 Je me permets de renvoyer ici à mon mémoire de DEA en philosophie: Antoine TINE, Le destin libéral de l'État de Droit: Schmitt ou Rawls ? Le choc de deux inconciliables, Paris, Université de Paris IV-Sorbonne, 1996.

52 Il convient ici de faire ici une mise au point. Une lecture rapide peut donner l'idée que Schmitt est hostile à toute forme de pluralisme du moment qu'il s'oppose à la discussion libérale. Ce que Schmitt critique, c'est le pluralisme absolu qui n'admet aucune restriction, sinon celle que fournit l'accord sur un ensemble de procédures et qui rejette toute référence à l'unité politique. Un tel pluralisme, pense t-il, participe de l'illusion formaliste de la neutralité de l'État de droit et ignore que tout pluralisme doit faire appel à l'unité, au risque de tomber dans la dissolution anarchiste du lien social. Cette conception de l'unité politique n'empêche pas Schmitt d'admettre le principe de la pluralité sociale. Bien que l'État soit le principe et le fondement de l'unité, il doit être soumis à une "éthique pluraliste ": "L'unité de l'État a toujours été une unité faite de multiplicité sociale. A diverses époques et dans différents États, elle a été très diversifiée, mais toujours complexe et, en un certain sens, en elle-même pluraliste. " : Carl SCHMITT, Parlementarisme et démocratie, trad. 
moderne en dépend. Mais aussi, c'est autour du pluralisme que se joue l'enjeu du politique. Il met en garde contre les points aveugles d'un certain type de pluralisme, celui de l'individualisme libéral selon lequel chaque individu est libre de participer à de multiples associations, dont aucune ne peut avoir priorité sur les autres et exiger de lui une obligation spéciale. Dès lors, estime Schmitt, le libéralisme met en cause l'existence d'une vérité absolue et «renonce à un résultat définitif $» 53$.

La pensée libérale implique que la vérité procède du libre arbitre des opinions et dissout la vérité dans le choix individuel ou le libre jeu de la discussion infinie. Selon les libéraux, tous les hommes peuvent découvrir la vérité, à condition qu'ils laissent de côté leurs intérêts afin de juger uniquement du point de vue de la raison. Cette position est celle du rationalisme des Lumières du XVIIIè siècle. De plus, en logeant l'État à la même enseigne que les associations religieuses et les groupements professionnels et en attribuant à l'individu un rôle déterminant dans la résolution des conflits, le libéralisme élude ou ignore la nature du phénomène politique :

« faire figurer, écrit-il, à la manière pluraliste, une association politique à côté d'une association religieuse, culturelle, économique ou autre, et la faire entrer en concurrence avec elles, cela n'est possible que pour autant que la nature du politique n'est pas prise en considération » 54 .

Ainsi donc, le libéralisme peut se mouvoir dans la reconnaissance équivoque de sphères hétérogènes : la morale et l'économie, l'esprit et les affaires, la culture et les affaires 55 En fait de reconnaissance, il s'agit plutôt d'un pis-aller qui conforte chaque individu dans sa propre vision, puisque les questions concernant les valeurs susceptibles d'être

par Jean-Louis Schlegel. Préface de Pasquale Pasquino, Paris, Seuil, 1988, p. 141.

53 Carl SCHMITT, Parlementarisme et démocratie, op. cit., p. 45.

54 CARL SCHMITT, La notion de politique. Théorie du partisan, trad. par M.L. Steinhauser. Préface de Julien Freund, Paris, Calmann-Lévy, 1972, p. 86.

55 Carl SCHMITT,. La notion de politique, op. cit., p.117. 
sources de conflits sont renvoyées à la sphère privée. C'est de cette façon, pense Schmitt, que le libéralisme fait l'impasse sur la dimension conflictuelle de la vie sociale et la permanence de l'antagonisme. Pour la pensée libérale, il n'existe pas de vérité absolue. Néanmoins un accord général peut résulter du libre jeu des intérêts privés ou d'une discussion rationnelle. Cet accord consiste en un ensemble de règles générales de procédure.

L'État de droit sert à cette fin. Schmitt s'en prend vivement à cette conception en lui reprochant, outre de nier le politique, de se nourrir de surcroît de l'illusion de la neutralité de l'État. Alors, tombe invariablement l'objection têtue selon laquelle le critère du politique, sa differentia specifica (selon l'arbre de Porphyre), c'est la relation ami/ennemi; autrement dit, la politique concerne la création d'un « nous » opposé à un " eux » et se situe donc dans le champ des identités et/ou identifications collectives. C'est dans la mesure où la politique est puissance de discrimination qu'elle peut créer de l'identité. En effet, l'identique ne se reconnaît comme tel que par rapport à ce qui ressemble. C'est dire que la politique s'appuie sur une homogénéité. C'est par ce biais qu'elle peut assurer une unité substantielle de la pluralité.

On le voit, à travers le rejet du pluralisme libéral, Schmitt refuse ce qui est constitutif de l'émergence de la modernité politique: l'abandon d'une vision substantialiste de la vérité et du bien commun. Schmitt n'accepte pas la "dissolution des repères de la certitude » 56 et le caractère contingent ou indéterminé de la démocratie. Il se place donc d'un point de vue pré-moderne, conservateur et autoritaire, qui méconnaît l'hétérogénéité de la démocratie moderne. Schmitt a une conception identitaire de la démocratie.

Sans homogénéité, pas de démocratie. Une véritable démocratie, selon Schmitt, doit reposer sur le fait que les individus sont sembla-

56 Claude LEFORT, Essais sur le politique, Paris, Seuil, 19 , p. 29. 
bles et sur la croyance que ce qui les maintient ensemble, c'est l'existence de l'État souverain. Cette instance est l'expression de la volonté générale ou de l'unité politique, laquelle ne peut dépendre de procédures relativistes de discussion. C'est l'État qui est la substance commune à laquelle participent les citoyens et qui les fait se reconnaître comme des semblables :

«Toute démocratie véritable repose sur le fait que non seulement ce qui est semblable reçoit un traitement semblable, mais encore - conséquence inévitable - que ce qui est non semblable ne jouit point d'un traitement semblable. Dans la démocratie entre donc nécessairement comme ingrédient pour commencer l'homogénéité, et ensuite, si besoin est, la mise à l'écart ou l'exclusion de l' hétérogène » 57 .

Comment concevoir cette homogénéité ? Dans la Théorie de la Constitution, Carl Schmitt rapporte l'homogénéité (Gleichartigkeit) à la notion d'égalité (Gleichheit) ${ }^{58}$. Cette égalité doit être considérée comme une égalité politique basée sur l'appartenance à une communauté politique selon des critères de race, de religion, de nationalité, de tradition culturelle, de foi commune... Il observe que depuis le $\mathrm{XIX}^{\mathrm{e}}$ siècle, c'est l'appartenance à une nation déterminée qui constitue la substance de l'égalité démocratique. Une citoyenneté démocratique se construit quand les membres de la collectivité politique peuvent se reconnaître comme appartenant à une même "communauté de destin", s'articulant autour de principes politiques de base (liberté, égalité). Cette égalité démocratique n'est valable que pour les nationaux, à l'intérieur d'un État :

«L'égalité qui fait partie de l'essence même de la démocratie ne s'applique donc qu'à l'intérieur (d'un État) et pas à l'extérieur: au sein d'un État démocratique tous les nationaux sont égaux. Conséquence pour

57 Carl SCHMITT, Parlementarisme et démocratie, op. cit, pp. 105-106.

58 Carl SCHMITT, Théorie de la Constitution, trad. par Lilyane Déroche. Préface d'Olivier Beaud, Paris, P.U.F., 1993. Pour une analyse de la conception schmittienne du droit, de la politique et de la démocratie, on consultera : Carlos - Miguel HERRERA (dir.), Le Droit, le Politique autour de Max Weber, Hans Kelsen, Carl Schmitt, Paris, L'Harmattan, 1995. 
le point de vue politique et celui du droit public : celui qui n'est pas citoyen n'a rien à faire avec cette égalité démocratique. » 59

C'est dire que la démocratie n'exclut pas l'absence de discrimination entre les hommes. L'égalité démocratique repose sur l'identité d'un peuple avec lui-même, à l'exclusion de l'hétérogène ou de l'étranger. En ce sens, l'État démocratique ne repose pas sur un contrat social, mais sur une égalité nationaliste. L'inégalité est le corollaire indispensable de cette égalité politique. Un peuple se pose en s'opposant à ce qui lui est étranger. D'où, selon Schmitt, la légitimité de l'exclusion :

«La force politique d'une démocratie se manifeste à sa capacité d'écarter ou de tenir éloigné l'étranger et le non-semblable, celui qui menace l'homogénéité. » 60

Assurément, la critique schmittienne de l'État de droit moderne est brillante et vigoureuse, mais assez décapante. Il est difficile d'en faire fi et de continuer à penser que l'avènement de la démocratie libérale et représentative constitue "la fin de l'histoire" 61 ou le nec plus ultra de l'évolution idéologique de l'humanité. Dans Parlementarisme et démocratie, Schmitt relève, d'une façon intransigeante, mais non moins perspicace, quelques points aveugles de l'État de droit libéral, censé fonctionner selon un système de représentation parlementaire.

Il défend l'idée que l'État de droit moderne appartient à l'univers idéologique du libéralisme représentatif. Or, pense Schmitt, le libéralisme nie la démocratie et vice-versa. Selon lui, la dimension représentative ne traduit pas une logique démocratique, mais est antidémocratique dans la mesure où elle rend impossible l'identité entre gouvernants et gouvernés. Schmitt pense la démocratie en tant que forme

59 Carl SCHMITT, Théorie de la Constitution, op. cit., p. 365.

60 Carl SCHMITT, Parlementarisme et démocratie, op. cit., p. 106.

61 Voir Francis FUKUYAMA, La fin de l'histoire et le dernier homme, trad. de l'anglais par Denis - Armand CANAL, Paris, Flammarion, 1992. 
d'État à "constitution mixte" à partir du principe de la représentation et du principe de l'identité.

Cette conception schmittienne de la démocratie comme identité ou homogénéité me paraît intenable et contradictoire avec la place éminente qu'il donne au souverain dans l'ordre politique. Du reste, la pensée de Schmitt est peu claire, puisque en même temps qu'elle affirme que la démocratie consiste dans l'identité de la loi et de la volonté populaire ou entre les gouvernants et les gouvernés, elle considère que la démocratie est compatible avec un régime autoritaire avec un "État qualitativement total". Il y a sans doute ici une réminiscence hégélienne ${ }^{6}$ qui achève de rendre la définition schmittienne de la démocratie, et partant sa critique de l'État de droit moderne, sujette à caution.

En outre, estimer comme Schmitt le fait que la substance de la démocratie consiste dans l'homogénéité nationale des membres du groupe politique - État, n'est-ce pas légitimer à peu de frais l'exclusion sur la base de critères comme la race, la nationalité, la langue...? N'est-ce pas une porte grande ouverte à la xénophobie, pire au racisme... ? La pensée de Schmitt se révèle là inconsistante et incapable de prendre en considération le multiculturalisme et la pluralité des nationalités à l'intérieur d'un État. Pasquale Pasquino peut écrire :

«(...) de plus en plus, notre horizon politique est celui des États plurinationaux. De sorte que "l'homogénéité nationale" comme fondement de nos démocraties paraît aujourd'hui, autant, sinon plus périmée que le parlementarisme de Condorcet. Elle ne peut réapparaitre que comme un mythe de mauvais aloi, un revenant au visage hideux » 63

Je pense que la conception schmittienne de la démocratie en tant que homogénéité présente néanmoins l'intérêt d'avoir fait voir qu'une

62 Voir : Jean - François KERVEGAN, Hegel, Carl Schmitt. Le politique entre spéculation et positivité, Paris, P.U.F., 1992.

63 P. PASQUINO, Préface à Parlementarisme et démocratie, op. cit., p. 18. 
démocratie ne peut être vivable et gouvernable sans l'existence d'une unité et de l'égalité politique. Cela est important. Mais, là où le bât blesse, c'est quand Schmitt considère cette base comme une substance rigide et comme un instrument d'exclusion de la différence. Cette vision substantialiste de la démocratie évacue d'ailleurs, d'une certaine manière, la question du pluralisme que le libéralisme avait fort justement problématisée.

Dans cette optique, la thèse schmittienne de la contradiction insurmontable entre le libéralisme et la démocratie manque de rigueur. On peut même dire que le sens spécifique de la modernité libérale - le pluralisme modéré - échappe à Carl Schmitt. Il rate, ce faisant, la véritable portée de l'État de droit en le réduisant à tort à n'être qu'une sécularisation de la théologie ${ }^{64}$. Son antilibéralisme militant le conduit à des positions conservatrices et totalitaires du politique. Certes, le libéralisme ne peut pas penser le politique comme il l'entend, mais estce à dire que le libéralisme se moque de toute politique à base de conflit et d'antagonisme social? C'est aller trop vite en besogne que de figer le politique dans la guerre et de penser que le pluralisme libéral serait irénique. Le libéralisme n'est pas un contrat social paisible garantissant sans coup férir un consensus absolument rationnel. Le pluralisme libéral est un pluralisme agonistique, car il ne saurait rester indifférent aux contradictions de la société.

Aussi paradoxal que cela puisse paraître, le pluralisme démocratique représente une difficulté face à la tentation hégémonique des partis politiques. Pourtant, c'est le pluralisme qui fonde l'être partisan. Mais, l'institutionnalisation partisane du pluralisme tend à figer les opinions et à réduire la portée du « fait du pluralisme ». Le pluralisme démocratique dépasse la configuration partisane et n'est pas équivalent à la simple arithmétique des partis politiques :

64 Voir : Carl SCHMITT, Théologie politique, 1922, 1969, trad. par Jean - Louis Schlegel, Paris, Gallimard, 1988. 
« (...) tantôt analysé comme instrument de division d'un politique unifié, tantôt compris comme négation de la pluralité du politique, le parti est dans tous les cas placé aux origines d'une question qui pourtant le dépasse. Il est l'impensé de la démocratie parce que le pluralisme lui-même est l'impensé du politique» 65 .

Il faut prendre garde d'exagérer cette contradiction ou de s'en offusquer en faisant du système des partis un lieu de dénégation du pluralisme. N'est-il pas plus juste de considérer que cette contradiction, plutôt que d'être négation absolue du pluralisme, est constitutive du politique ? La contradiction réside en ce que le politique demeure tendu entre deux exigences, l'unité et la pluralité, l'identité et la différence, le plein et le vide. De là le paradoxe du politique. Le pluralisme suppose l'exercice de la libre concurrence des idées et des représentations pouvant mener au "polythéisme des valeurs » ${ }^{66}$, des choix et des identités partisanes. L'idée du pluralisme se présente comme un fondement de la démocratie. Pourtant, au même moment elle affirme que rien n'est fondamental; elle se pose en s'opposant à elle-même. L'argument du pluralisme, l'absence du fondement, peut-il être appliqué à l'idée même du pluralisme ? Le pluralisme ne serait-il pas, en son principe, auto-contradictoire ou en tout cas ambigu?

Il est vrai que le pluralisme est non seulement en butte à toutes sortes de fondamentalismes, mais peut lui-même devenir un fondamentalisme. Le pluralisme et la recherche du fondement de l'action appartiennent à la même matrice ${ }^{67}$. L'opposition pluralisme-monisme est

65 J.-M. DONEGANI et M. SADOUN, La démocratie imparfaite. Essai sur le parti politique, Paris, Gallimard, 1994, p.183.

66 Max WEBER, Le savant et le politique, trad. fr. J. Freund, avec une préface de R. Aron, Paris, Plon, 1959, p. 83 et sqq; Cf. Sylvie MESURE et Alain RENAUT, La guerre des dieux. Essai sur la querelle des valeurs, Paris, Grasset, coll. « Le collège de philosophie », 1996, pp. 45-59.

67 Cf. Louise MARCIL-LACOSTE, « The Paradoxes of pluralism », in Chantal MOUFFE (ed.), Dimensions of radical democracy: pluralism, citizenship, community, London, Verso, 1992, pp. 128-142 ; Voir: William E. CONNOLLY, The ethos of pluralization, Minneapolis, University of Minnesota Press, 1995. 
de ce point de vue sujette à caution. Comme le dit très bien Jean Leca :

« De fait, au moment où la démocratie semble devenir pluraliste, le pluralisme s'affirme si radicalement qu'il prend une petite allure moniste : tout doit lui être subordonné. Or c'est un hommage à rendre au pluralisme que de constater qu'aucune valeur, étant toujours socialement instituée, ne peut être pure, absolue et univoque, et ceci vaut aussi pour le pluralisme, comme pour la démocratie $» 68$.

Mais, ce qui est rejeté à travers l'exigence de la pluralité, ce n'est pas en soi l'idée de l'unité ou celle du fondement, mais une certaine dogmatisation du fondement, qui consisterait à dire qu'il y a un seul principe du croire et du savoir comme dans « l' État théologien » que dénonce à juste titre Achille MBEMBE 69.

Pour une vue plus modérée, nous proposons d'articuler la recherche du pluralisme à l'existence de pôles de consensus politique, signes de réussite de procédures de légitimation et d'adoption de règles publiques d'action. Une société politique ne peut se réaliser dans la confusion chaotique des identités partisanes et la "cacophonie des propagandes » ${ }^{70}$. La reconnaissance de la pluralité des allégeances partisanes n'est pas une absolutisation outrancière des différences politiques. Un pluralisme non régulé peut exposer la société au syndrome de Babel ou à un individualisme atomistique.

La démocratie n'est pas réductible à l'unique principe pluraliste. L'invention démocratique de la modernité ne réside pas dans la seule démarche agonistique, c'est-à-dire dans la mise en évidence de ce qui, dans la société, est facteur de conflit, de lutte. Une démocratie viable

68 Jean LECA, «La démocratie à l'épreuve des pluralismes », in Revue Française de Science Politique, (46), 2, avril 1996, p.

69 Achille MBEMBE, Afriques indociles. Christianisme, État et Pouvoir en Société Postcoloniale, Paris, Karthala, 1988, p.

70 Paul VALADIER, Agir en politique. Décision morale et pluralisme politique, Paris, Cerf, 1980, p. 64. 
ne se nourrit-elle pas aussi de la négociation de compromis, de sphères de consensus? Quelle forme de consensus peut alors permettre une démocratie viable? Y a t-il compatibilité ou non entre la recherche de consensus et le fait du pluralisme?

Pour élucider ce problème précis, nous pouvons recourir à la philosophie politique de Jürgen Habermas. Héritier du siècle des Lumières et de la "Théorie critique » (Ecole de Francfort) ${ }^{71}$, Habermas propose un ambitieux projet de défense de la modernité (« die Moderne »). Il cherche alors à reconsidérer les potentialités émancipatrices de la démocratie à travers une théorie du consensus par la libre discussion. Mais, c'est surtout en s'inspirant de la théorie des actes de langage (Austin, Searle, Strawson, Grice) présentée comme une «pragmatique universelle » que Habermas va formuler une conception de la démocratie comme communication et discussion dans un espace public. La rationalité communicationnelle 72 de la démocratie recherche

71 On consultera: M. JAY, L'imagination dialectique. L'école de Francfort: 1923-1950, Paris, Payot, 1989 ; R. WIGGERSHAUS, L'Ecole de Francfort. Histoire, développement, signification, Paris, PUF, 1993 ; Jean-Godefroy BIDIMA, Théorie critique et modernité négro-africaine. De l'Ecole de Francfort à la "Docta Spes africana", Paris, Publications de la Sorbonne, 1993 ; F. VANDERBERGHE, Une histoire critique de la sociologie allemande. Aliénation et Réification. Tome II : Horkheimer, Adorno, Marcuse, Habermas, Paris, La Découverte/ Mauss, 1998.

72 Il y a quatre modalités d'agir chez Habermas : l'agir régulé par des normes, l'agir expressif, l'agir stratégique et l'agir communicationnel. Retenons ici la distinction qu'Habermas fait entre "agir communicationnel" et "agir stratégique" : "L'agir communicationnel se distingue (...) de l'agir stratégique en ce qu'une coordination réussie des actions ne s'appuie pas sur la rationalité finalisée des plans d'action chaque fois individuels, mais sur la force rationnellement motivante d'efforts entrepris en vue de l'entente, et donc en ce qu'elle s'appuie sur une rationalité qui se manifeste dans les conditions appropriées à un accord obtenu par la communication." (J. HABERMAS, La pensée postmétaphysique, Paris, Armand Colin, 1993, p.72 ; Cf. aussi du même auteur Théorie de l'agir communicationnel, 2 tomes, trad. de l'allemand par JeanLouis Schlegel, Paris, Fayard, 1987 ; Morale et Communication. Conscience morale et activité communicationnelle, trad. et introd. par $\mathrm{C}$. Bouchindhomme, Paris, Cerf, 1986 p.79). 
l'entente et l'accord entre des sujets capables d'agir et de parler en vue d'une action commune.

Mais, Habermas ne cède $\mathrm{t}$-il pas à « l'illusion transcendantale » du consensus et de la communication réussie ? Un mythe du dialogue ? Si le sujet n'est pas transparent à lui-même et à fortiori aux autres, comment l'activité communicationnelle peut-elle être le lieu de la «vérité consensuelle»? Que peut même cacher et révéler l'idée de vérité consensuelle ? L'adjectif « consensuelle » n'est-il pas un qualificatif abusif? N'y a t-il pas risque de figer ainsi le concept de vérité et de faire l'impasse sur le caractère provisoire, transitoire et nomade de toute réalité en gestation?

$\mathrm{Au}$ fond, la vérité ne saurait être ni l'idole, ni l'appropriation commune d'un consensus rationnel. Même et surtout dans un espace public démocratique, on ne peut parvenir à une vérité consensuelle ou à l'intercompréhension mutuelle. En effet, la discussion achoppe ici sur l'opacité invincible de la communication. Il y a toujours plusieurs niveaux de langage et d'interprétation qui résistent à la réduction pragmatique. Habermas ne tient pas assez compte de cette difficulté, inhérente à ce que Paul Ricoeur appelle le «conflit des interprétations $\gg$.

En fait, Habermas n'est-il pas pris comme Gadamer dans une métaphysique de la communication présupposant la propriété d'un sens, d'un sens qui pré-approprie l'un à l'autre les interlocuteurs de la discussion argumentative ? Jean-Luc Nancy ne pense t-il pas juste en disant que la théorie du consensus raisonnable de Habermas rejoint en partie l'herméneutique de Gadamer -dialogue comme vérité- qu'elle critique par ailleurs. Le projet de la modernité, tel qu'il est réactivé par Habermas, repose sur un acte de « confiance anthropologique » en 
la possibilité d'une réconciliation entre conscience critique et identité 73 .

L'éthique habermassienne de la discussion ne se donne-t-elle pas pour ressource la capacité sociale et communicationnelle de fonder des identités collectives ou de se les réapproprier? Pourtant, que les identités sont difficiles! Par sa visée d'une communication sans contrainte et préservée de la violence, Habermas ne tombe t-il pas dans l'illusion du consensus? Il sait cependant que le consensus n'est jamais donné à « l'état pur », ni atteint comme une « donnée immédiate de la conscience » (Henri Bergson), ni même comme une "fin de l'histoire » (Francis Fukuyama). D'où la dimension contrefactuelle de la théorie de l'agir communicationnel. Chez Habermas, le consensus est pensé sur le mode d'une approche asymptotique à l'idée régulatrice d'une raison communicationnelle et productrice, grâce à l'argumentation, de vérité. Il y a, inséparablement, chez Habermas le goût du consensus réconciliateur et le sens de l'irréconciliation et de la rupture.

Mais tout de même, cette théorie de la vérité consensuelle tend à faire obstacle à l'antagonisme des intérêts de classes et occulte la part

73 Une nuance: la pensée de Habermas n'est pas aussi métaphysique qu'elle pourrait le paraître à première vue. Elle se veut d'ailleurs post-métaphysique. Il y a, en fait, un contraste entre les écrits théoriques de Habermas sur le consensus et l'agir communicationnel et ses textes polémiques contre Heidegger et l'idéalisme allemand, notamment à propos de la «Querelle des Historiens » (Historikerskeit) de 1986 ( Profils philosophiques et politiques, Paris, Gallimard, 1981). Il y a chez Habermas un sens de l'irréconciliation et de la rupture. Cela peut se voir dans sa réflexion sur l'Allemagne, sur l'identité politico-culturelle allemande, marquée par le «moment » d'Auschwitz, taraudée par le mémoire des crimes nazis, la mémoire de la Shoah juive. Rendant compte de cette situation, Edouard Delruelle écrit par exemple : "L'un des enjeux de la pensée habermassienne, mais peut-être aussi l'une de ses contradictions est de tâcher de se rendre fidèle à ce qu'il appelle le "regard des exilés » (...). Mais ce regard des exilés, ne fait-il pas échec au présupposé de l'entente, de la réconciliation qui domine le schéma habermassien du consensus ? » : Le consensus impossible, Bruxelles, Ousia, 1993, p.13-14). 
non moins négligeable de la violence dans l'interaction sociale et politique. Est-ce à dire que Habermas ne pense suffisamment le pluralisme ? De prime abord, il semble difficile de dénicher une théorie du pluralisme chez Habermas, tellement la recherche du consensus est une exigence forte qui couvre d'insignifiance la diversité. L'altérité ou la pluralité des positions sociales est posée comme tremplin pour l'activité communicationnelle?

Toutefois, Habermas ne conçoit pas le point de vue moral en dehors du contexte des interactions médiatisées par le langage, traversées par les conflits et les distorsions qui rendent la communication à autrui si peu transparente. Habermas n'est donc pas dupe de son exigence consensuelle, car pour lui la communication naît précisément de l'absence d'entente. Et, l'universel visé n'est pas un point de vue de Dieu, mais un point de vue du «nous » qui suppose l'existence plurielle des perspectives de vie et la liberté des protagonistes de l'argumentation. Mais là où le bât blesse, c'est quand Habermas déploie toute sa théorie à reconstruire une communication sans conflit à partir de la « situation idéale de parole ». N'opère-t-il pas là une absorption du pluralisme dans l'objectif quasi transcendantal d'un « discours universel $» ?$

D'ailleurs, le concept d'universel demeure chez lui assez problématique. N'est-il pas fondé sur une conception de la rationalité et du discours tels qu'ils sont élaborés en Occident ? Dès lors, l'universalité de Habermas n'est-elle pas ethnocentrique ? Elle ne tient pas compte de la diversité impliquée dans les cultures humaines au regard des problèmes de la communication inter (multi) culturelle. N'oublie-t-il pas que c'est au cœur même du particulier, dans le respect des différences et de la diversité, que s'inscrit la recherche de l'universel ? Vincent Descombes a raison, me semble t-il, de reprocher à Habermas de négliger la multiculturalité et la relativité culturelle :

« Habermas ne s'avise pas qu'il donne la parole à une tradition nationale particulière quand il hégélianise de façon si décidée. Un sociologue aurait 
plus facilement reconnu que la conscience philosophique du fait moderne avait trouvé différentes expressions selon les cultures nationales. (...) Le philosophe n'hésite pas à parler au singulier du projet moderne de rationalisation. Du point de vue d'une analyse sociologique, la dynamique qui constitue pour nous le procès de modernisation du monde est la résultante d'un jeu complexe d'échanges entre des sociétés porteuses de cultures distinctes. » 74

Pourtant, en un certain sens, Habermas fait bien d'insister sur l'idéal consensuel. En effet, une société politique ne peut se maintenir sans certaines procédures de communication et des sphères publiques de consensus. Pour vivre ensemble, les hommes ne peuvent faire autrement que d'adhérer à des valeurs communes et à des règles normatives qui constituent les fondements de la légitimité.

Les institutions sociales et politiques tiennent dans la mesure où elles mobilisent une réelle participation aux pratiques sociales qui tissent tant la trame de l'État que de la société civile. La vie politique suppose qu'il y ait accord sur les fondements de la coexistence sociale, malgré l'antagonisme des perspectives. Mais l'exigence du consensus n'est pas exclusive de la réalité du pluralisme. La vie politique démocratique, pour préserver la discussion et ne pas livrer les citoyens au caprice de l'arbitraire, doit reposer sur la tension entre consensus et pluralisme.

Penser le pluralisme politique, c'est penser la transformation de l'ennemi inconnu en adversaire, c'est vouloir passer de l'antagonisme décisionniste (Max Weber, Carl Schmitt) 75 à un agonisme procédural. La politique ne consiste-t-elle pas à chercher à désamorcer (non à supprimer) la puissance de la violence qui accompagne toujours les constructions d'identités collectives ? Dans cette entreprise, il ne s'agit pas de domestiquer l'inimitié en jetant tout son dévolu sur la

74 Vincent DESCOMBES, Philosophie par gros temps, Paris, Minuit, 1989, p.53.

75 Voir Sylvie MESURE et Alain RENAUT, La guerre des dieux. Essai sur la querelle des valeurs, Paris, Grasset \& Fasquelle, 1996. 
généreuse grâce de l'amitié ou de l'entente mutuelle, mais en créant des institutions et des procédures capables d'assurer un "modus vivendi », grâce à des règles de jeu favorisant le respect des identités plurielles. C'est dans ce sens, par exemple, qu'Elias Canetti analyse l'institution du vote en démocratie comme un renoncement à tuer pour s'en remettre à l'opinion du plus grand nombre 76 .

En mettant entre parenthèses l'agir stratégique, entaché de violence et marqué par les intérêts de classe au profit de l'agir communicationnel, censé s'appuyer sur les présupposés de l'intercompréhension, Habermas ne néglige t-il pas la question du pluralisme politique ? En s'imaginant qu'il est possible d'atteindre un consensus résultant de la discussion, en « situation idéale de parole », Habermas ne court-il pas le risque de congédier les questions proprement politiques de la dynamique agonistique, de la violence, du pouvoir et de la signification de l'adversaire. Certes, une société politique se nourrit du dialogue, de la délibération publique rationnelle, de la négociation de compromis, donc du consensus.

Mais cette exigence de consensus ne saurait être indifférente aux différences, c'est-à-dire aux espaces d'hétérogénéité ou de « dissensus ». Pour penser juste, il faut situer la dynamique agonistique $77 \mathrm{du}$ politique entre le consensus et le pluralisme. Un État démocratique est un espace public de contrôle qui ne se propose pas d'éradiquer les conflits, mais d'inventer des procédures leur permettant de s'exprimer et de rester négociables. Il doit accueillir les conflits et les trancher (fonction judiciaire) tout en gardant une visée de réconciliation. Paul Ricoeur parle, dans ce sens, de « consensus conflictuel » 78 .

76 Cf. Elias CANETTI, Masse et puissance, Paris, Gallimard, 1966.

77 -agonistique vient du grec $\alpha \gamma \omega v$ qui signifie «lutte, combat, débat»; . $\alpha \gamma \omega v \imath \zeta o \mu \alpha \imath=$ lutter ou être l'objet d'une lutte, d'un débat, $\alpha \gamma \omega v \imath \sigma \tau \imath \kappa o \zeta:$ « qui concerne la lutte ». C'est dire que le pluralisme suppose l'existence de conflits, de tensions ou au moins d'un débat dans la vie sociale.

78 Cf. Paul RICOEUR, Du texte à l'action. Essais d'herméneutique II, Paris, Seuil, 1988. 
Cela a déjà été dit: une société politique ne peut se maintenir sans certaines procédures de communication établissant des sphères publiques de consensus. Le consensus est nécessaire pour conjurer le danger du relativisme des valeurs pouvant conduire à la dissolution anarchique. C'est pourquoi, Habermas voit juste en portant son effort sur l'élucidation des principes moraux autour desquels s'articule la communication intersubjective.

Pour vivre ensemble, les hommes - s'ils le veulent-, peuvent-ils faire autrement que d'adhérer à des valeurs communes et à des règles pragmatiques et normatives régissant leurs pratiques? Et les institutions ne tiennent-elles pas dans la mesure où elles requièrent la réelle participation des citoyens aux pratiques sociales qui tissent aussi bien la trame de l'État que de la société civile? ÀA moins que l'espace public ne soit livré au jeu de la décision arbitraire du «Souverain » ou du « Prince ».

Toutefois, la vie politique, en démocratie surtout, ne saurait être réduite à l'exigence du consensus. Il est des moments et des espaces rebelles au consensus commun et universel. Le trop-plein de consensus abolit la conflictualité politique et peut même se retourner en son contraire. Il faut lutter contre la boulimie régulatrice et conciliatrice, car l'espace politique est essentiellement marqué par une sorte d'hétérogénéité irréductible, de faille indocile, en quoi résident précisément la liberté et les appels d'indiscipline. Le pluralisme démocratique se situe dans cet entre-deux ambigu, entre la règle et l'indomptable. Comme tel, il n'est ni l'expression débridée de soi et de l'anarchie, ni le refus systématique de l'unité politique.

C'est dire que le pluralisme et le monisme, l'un et le multiple ou l'autre et le même, loin d'être des notions absolument incompatibles, devraient être pensés comme deux exigences corrélatives, deux pôles dialectiques. Sans l'un de ces deux éléments, il n'y a pas de réelle situation de démocratie, mais soit un régime de domination totalitaire 
reposant sur l'homogénéité de la pensée unique, soit une politique libertaire et relativiste dépourvue de projet de vivre ensemble. D'un côté, l'on aboutit à l'embrigadement des libertés, de l'autre à la dissolution anarchiste du lien social.

La démocratie ne met donc pas fin aux conflits et ne se conduit pas irrémédiablement au consensus. Elle peut, tout au plus, atténuer et contenir les conflits dans des limites de compromis raisonnables. N'est-ce pas le conflit qui nourrit le pluralisme et fait de la démocratie une expérience fragile, incertaine, provisoire et imparfaite ? Le conflit est un phénomène intraitable dans une société. Son existence est le signe de la reconnaissance du « tiers » ou de la liberté.

La démocratie ne repose-t-elle pas sur le vide dans la mesure où comme le dit Claude Lefort, elle est une « dissolution des repères de la certitude » ? Elle est confrontée en permanence à l'incertitude et à l'hétérogénéité des intérêts et des fins individuels. Il y a, au cœur de toute démocratie véritable, un je-ne-sais-quoi de rebelle à toute tentative de résolution dans un "savoir absolu », dans un "système » (Hegel), qu'on peut appeler en suivant Jean-François Lyotard le « différend », c'est-à-dire "l'état instable et l'instant du langage où quelque chose qui doit pouvoir être mis en phrase ne peut pas l'être encore ${ }^{79}$. Ce quelque chose, c'est une altérité irréductible, fondement du pluralisme démocratique, source des conflits sociaux et des crises politiques. Parce qu'elle s'appuie sur cette donnée, la philosophie est fondamentalement une épreuve de mise en crise permanente du schéma théologico-politique ou des régimes de la certitude.

Toutefois, dire cela ne signifie pas nécessairement un éloge du conflit, de l'incertitude et de l'instabilité. Une société désarmée face au conflit et confrontée d'une manière perpétuelle à un climat d'hostilité et de violence ne risque-t-elle pas de cesser d'être vivable et de se détruire? Il n'est pas question de soutenir le conflit pour le

79 Jean-François LYOTARD, Le différend, Paris Minuit, 1983. 
conflit. Cela ne servirait pas à l'épanouissement des libertés et d'un pluralisme responsable, encore moins du consensus. Il faut, à mon avis, prudence garder en tenant que le pluralisme politique implique certes une multiplicité d'héritages et de trajectoires historiques, mais aussi un devoir de sociabilité responsable. Dialectique négative, le pluralisme est une "possibilité d'accord par les désaccords », écrit Julien Freund 80.

Y a-t-il, à vrai dire, accord plus fondateur de la quête et de l'invention démocratiques que celui qui encourage, reconnaît et tente de dépasser les désaccords et les conflits? La recherche du consensus ne se trouve t-elle pas dans un travail de transformation du dissensus ou de l'hétérogénéité sociale et politique ? Le consensus ne saurait rester indifférent aux appels du pluralisme, parce que la vie politique

« vise à constituer un_nous dans un contexte de diversité et de conflit. Or, (...) pour construire un nous, il faut le distinguer d'un eux. C'est pourquoi la question cruciale d'une politique démocratique n'est pas d'arriver à un consensus sans exclusion (...) mais de parvenir à établir la discrimination nous/eux d'une manière qui soit compatible avec le pluralisme » 81

80 Julien FREUND, L'Essence du politique, Paris, Sirey, 1986 (1965), p.210.

81 Chantal MOUFFE, Le politique et ses enjeux. Pour une démocratie plurielle, Paris, La Découverte/ Mauss, 1994, p.13. 


\section{BIBLIOGRAPHIE}

$\underline{\text { Retour à la table des matières }}$

ARENDT, Hannah, Qu'est-ce que la politique ?, Paris, Seuil, 1995.

AUDARD, Catherine, "Pluralisme et consensus : une philosophie pour la démocratie ? », Critique, n 505-506, juin-juillet 1989

BAYART, Jean- François.- L'illusion identitaire.- Paris : Fayard, 1996.

BERLIN, Isaiah, Les penseurs russes, Albin Michel, 1984

BIZEAU, Jean-Pierre.- «Pluralisme et démocratie ».- RDP et de la science politique en France et à l'étranger.- Avril 1993, pp. 513-542.

CANAVAN, Francis.- The pluralist game : pluralism, liberalism and the moral conscience.- Lanham, Md.: Rowman and Littlefield, 1995.

CHATELET, François, DUHAMEL, Olivier, PISIER, , Evelyne, Dictionnaire des oeuvres politiques, Paris, P.U.F, 1995, 106-123

COLE, George Douglas Howard ; FIGGIS, John Neville ; LASKI, Harold Joseph.- The pluralist theory of the state : selected writings.London : Routledge, 1989.

CONAC, Gérard (dir.).- L'Afrique en transition vers le pluralisme politique.- Paris : Economica, 1993.

CONNOLLY, William E.- The Bias of Pluralism.- New York: Atherton, 1960.

CONNOLLY, William.- The ethos of pluralization.- Minneapolis, Minn. : University of Minnesota Press, 1995.

DAHL, Robert.- Polyarchy: Participation and Opposition.- New Haven, Conn. : Yale University Press, 1971.

DAHL, Robert A.- Dilemmas of Pluralist Democracy. Autonomy vs Control..- New Haven and London : Yale University Press, 1982. 
DAHL, Robert A., Democracy and its critics, New Haven and London, Yale University Press, 1989.

DE CERTEAU, Michel, L'invention du quotidien. Tome 1 : Arts de faire, Paris, UGE, 1980.

DELRUELLE, Edouard, Le consensus impossible, Bruxelles, Ousia, 1993.

DONEGANI, Jean-Marie et SADOUN, Marc, La démocratie imparfaite. Essai sur le parti politique, Paris, Gallimard, 1994.

EBOUSSI BOULAGA, Fabien, Les conférences nationales en Afrique noire. Une affaire à suivre, Paris, Karthala, 1993.

FOUCAULT, Michel, Dits et Ecrits 1954-1988, Tome IV : 19801988, Paris, Gallimard, 1994.

FREUND, Julien, L'Essence du politique, Paris, Sirey, 1986, (1965).

GUEYE, Sémou Pathé, Du bon usage de la démocratie en Afrique. Contribution à une éthique et à une pédagogie du pluralisme, Dakar, Nouvelles Editions Africaines du Sénégal, 2003.

GRAZIANO, Luigi.- « Traditions in pluralist thought ».- in International Political Science Review.- vol. 17, nº , 1996, pp. 237- 344.

GRAZIANO, Luigi.- " Le pluralisme : une analyse conceptuelle et comparative ».- in Revue française de science politique.- vol. $46, \mathrm{n}^{\circ} 2$, avril 1996, pp. 195-224.

HABERMAS, Jürgen, Théorie de l'agir communicationnel, 2 tomes, trad. par J.-L. Schlegel, Paris, Fayard, 1987.

HAMILTON, A., MADISON, J., JAY, J., The Federalist Papers (1787), ed C. Rossiter, New American Library, 1961 ; trad. fr. : Le Fédéraliste, Paris, L.G.D.J., 1957.

HELD, David, Models of Democracy, Cambridge, Polity Press, 1987.

HOLLER, Manfred J. (ed), The Logic of Multiparty System, Dordrecht, Boston, Lancaster : Kluwer Academic Publishers, 1987.

KEKES, John.- The morality of pluralism.- Princeton, N.J., Princeton University Press, 1993. 
KINGWELL, Mark.- A civil tongue : justice, dialogue and the politics of pluralism.- University Park, Pennsylvania : State University Press, 1995.

KLIOT, Muriot ; WATERMAN, Stanley (eds).- Pluralism and political geography: people, territory and state.- London; Croom Helm ; New York : St Martin's Press, 1983.

KUPER, L. and SMITH, M.G.- Pluralism in Africa.- Los Angeles, 1971.

LECA, Jean, « Libéralisme, pluralisme, communautarisme : actualité d'Isaiah Berlin », in Commentaires, 70, 1995, pp. 369-382

LECA, Jean.- «La démocratie à l'épreuve des pluralismes ».- in Revue française de science politique.- vol. 46, $\mathrm{n}^{\circ} 2$, avril 1996, pp. 225-279.

MANIN, Bernard, Principes du gouvernement représentatif, Paris, Flammarion, 1996.

MARCIL-LACOSTE, Louise.- « The Paradoxes of pluralism ».- in Chantal MOUFFE (ed.).- Dimensions of radical democracy : pluralism, citizenship, community.- London : Verso, 1992.

MESURE, Sylvie et RENAUT, Alain, La guerre des dieux. Essai sur la querelle des valeurs, Paris, Grasset, 1996.

MILLER, David Leslie and WALZER, Michael (eds).- Pluralism, justice and equality.- Oxford : Oxford University Press, 1995.

MOON, Donald J.- Constructing community : moral pluralism and tragic conflicts.- Princeton, N.J., Princeton University Press, 1993.

MOUFFE, Chantal (ed.).- Dimensions of radical democracy : pluralism, citizenship, community.- London : Verso, 1992.

MOUFFE, Chantal.- Le politique et ses enjeux. Pour une démocratie plurielle.- Paris : La Découverte / Mauss, 1994. 175 p.

NDUE, Paul Ntungwe.- "Africa's turn toward pluralism ».- in Journal of Democracy, 5 (1), january 1994, pp. 45-54.

OOMEN, T. K.- " Contested boundaries and emerging pluralism ».- in International Sociology.- vol. 10, n 3, 1995, pp. 251- 268.

RAWLS, John, Théorie de la Justice. Trad. de l'anglais par Catherine Audard, Paris, Seuil, 1987. 
RAWLS, John, Justice et démocratie. Introduction, présentation et glossaire par Catherine Audard. Trad. de l'anglais par C. Audard, P. De Lara, F. Piron et A. Tchoudnowsky, Paris, Seuil, 1993.

RAWLS, John, Libéralisme politique. Trad. De l'anglais par C. Audard, Paris, PUF, 1995.

RESCHER, Nicholas.- Pluralism : against the demand for consensus.- Oxford : Clarendon Press, 1993.

REZLER, André.- Le pluralisme : aspects théoriques et historiques des sociétés ouvertes.- Genève / I.U.E. : Georg Eshel, 1990, $157 \mathrm{p}$.

RONEM, Dov (ed).- Democracy and pluralism in Africa.- Holder and Stoughton : Lynne Rienner Publishers, 1986.- 220p.

SARTORI, Giovanni.- The Theory of Democracy revisited.- Part one: The contemporary debate.- Chatham, New Jersey: Chatham House Publishers, Inc., 1987, pp. 39-181.

SCHMITT, Carl, La notion de politique. Théorie du partisan. Trad. par M.-L. Steinhauser. Préface de Julien Freund, Paris, Calmann-Lévy, 1972.

SCHMITT, Carl, Parlementarisme et démocratie, trad. par Jean-Louis Schlegel. Préface de Pasquale Pasquino, Paris, Seuil, 1988, p. 141.

SCHMITT, Carl, Théorie de la Constitution. Trad. par Lilyane Déroche. Préface par Olivier Beaud, Paris, PUF, 1993.

SEURIN, Jean-Louis (dir.).- La Démocratie pluraliste.- Paris : Economica, 1981.

SCHUMPETER, J. A., Capitalisme, socialisme et démocratie, Paris, Payot, 1951.

TENZER, Nicolas, Philosophie politique, Paris, PUF, $1998^{2}$.

VALADIER, Paul, Agir en politique. Décision morale et pluralisme politique, Paris, Cerf, 1980.

VEYNE, Paul.- Le pain et le cirque. Sociologie historique d'un pluralisme politique.- Paris : Seuil, 1976.

ZOLO, Danilo.- Democracy and Complexity : a realist approach.Transl. from the Italian by David Mckie.- Cambridge : Polity Press, 1992. 
WALZER, Michael, Spheres of justice : a defense of pluralism and equity.- Oxford : Robertson, 1983.

WALZER, Michael, Pluralisme et démocratie. Trad. Collective. Introduction de Joël Roman, Paris, Esprit, 1997.

WISEMAN, John A.- « Democracy and the new pluralism in Africa : causes, consequences and significance ».- in Third World Quaterly, vol. 14, n 3, 1993, pp. 439-449.

YOUNG, Crawford.- The Politics of Cultural Pluralism.- Madison, The University of Wisconsin Press, 1976.

\section{Fin du texte}

